\title{
Characterization of Fiber Bragg Grating Sensor in a High Speed Rotating Pipe
}

\author{
Rajinikumar Ramalingam, Holger Neumann \\ Karlsruhe Institute for Technology, Campus Nord, \\ Institute of Technical Physics, \\ Eggenstein-Leopoldshafen, Germany
}

\begin{abstract}
:
The next generation generators will use high temperature superconductors (HTS) for its rotor construction replacing currently used conventional copper rotor. This will enhance the generator efficiency to a steep increase of $0.5 \%$ which results in saving of $\$ 3.8 \mathrm{M}$ for $900 \mathrm{MVA}$ Generator according to calculation done in 2007. The HTS rotor, rotates at $3600 \mathrm{rpm}$ will be cooled by liquid Neon to $30 \mathrm{~K}$ using cooling channel arrangements. The temperature profile of the cooling channels has to be continuously monitored to ensure the sufficient cooling. But, it is a challenging task to identify a feasible sensing system and the associated signal transmission/processing unit. One of the possible sensing systems for the above described application could be Fiber Bragg Grating. Gratings with different spatial period are arranged at various positions along a single mode fiber in the HTS Generator. Rotor temperature will vary the gratings' periods, which can be read out with a tunable laser in a wavelength division multiplexing (WDM) scheme. The spectral position of the reflections may be correlated with the spatial position of the gratings, and the shift of the gratings' maximum reflection indicates the change of the gratings' period, which in turn measures temperature. In this paper, the initial experiments, which demonstrate the rotational effect on FBG sensor at room temperature is reported.
\end{abstract}

Key words: Fiber Bragg grating sensors, HTS Generator, rotating pipe.

\section{Introduction}

The cryogenics group at Institute of Technical physics (ITEP), Karlsruhe Institute of Technology, North campus (KIT-CN) is involved in developing and investigating a High Temperature Superconductor (HTS) based experimental basic model rotor along with its industry partner. This investigation results will later help in building and testing of planned 900 MVA High Temperature Superconductor (HTS) generator. HTS materials, especially Magnesium di boride $\left(\mathrm{MgB}_{2}\right)$ are used in many superconducting applications because of their low investment cost and higher critical temperatures compared with $\mathrm{NbTi}$ or $\mathrm{Nb}_{3} \mathrm{Sn}$. Apart from that the use of HTS in generators reduces mass and volume to about $70 \%$ of those of its conventional equivalent, provides high efficiency due to low loss and high dynamic performance. The trend of increase in the HTS generator efficiency is shown in Figure 1. The steep increase of $0.5 \%$ will results in the saving of approximately $\$ 3.8 \mathrm{M}$ for $900 \mathrm{MVA}$ generators [1]. The rotor of the HTS generator will be cooled by supply of liquid Neon (LNe) from the reservoir of the rotating cryostat. The conceptional design, construction and operation of such a rotating cryostat works on the thermosyphon cooling cycle principle at $30 \mathrm{~K}$ is a challenge for the developers.

The cryogenics group at ITEP has gained experience in constructing a cooling system for rotating apparatus using liquid helium ( $\mathrm{LHe}$ ) between 1977 and 1983 [2]. The principle of the thermosyphon within a rotating cryostat was investigated and some components like rotatable feed -through for $\mathrm{LHe}$ was developed. Also the procedure and construction for refilling of $\mathrm{LHe}$ into the rotor of a superconductive generator was patented [3]. With this broad experience, cryogenics group at ITEP mainly involves in solving different technical problems in developing, constructing and operating a thermosyphon cycle with Liquid Neon (LNe) in a rotatable cryostat. A better understanding of the 
fluid dynamics and the heat transfer characteristics within such a cycle is important. Based on the understanding of the heat transfer characteristics, the technical solution to control and optimize the flow has to be developed.

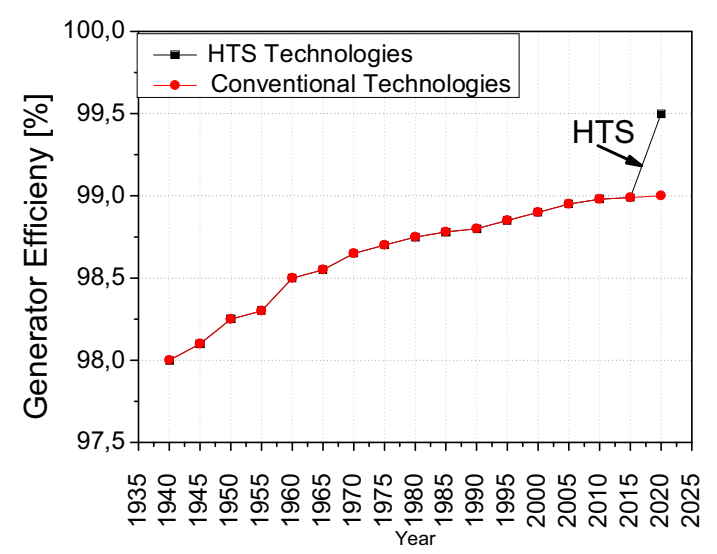

Fig.1. Trend comparison between the efficiency of conventional and HTS technologies [1]

To understand the fluid dynamics and the heat transfer characteristics within the thermosyphon cycle of a rotating cryostat, it is evident that a suitable measurement system should be identified

The planned HTS generator will rotate at the speed of $3600 \mathrm{rpm}$ and works at $30 \mathrm{~K}$. Hence the available conventional electro mechanical sensors like cernox, Russian TVO sensors or cheap platinum sensors (PT 10000) may be the choice. With the use of such sensors the mass flow shall be measured by two methods namely constant heating power and heat pulse.

In constant heating power method, the heater can be placed along the cooling duct which heats the outer ducts continuously. The coolant travels through the duct warms up and will rise the coolant temperature. The absolute temperature of the coolant can be then measured at various locations and hence very good absolute measurement accuracy is necessary between sensors. The mass flow rate could be then calculated by using the heating power, heat capacity of the coolant and the temperature rise. In the heat pulse method, a heat pulse will be applied at the outer surface of the cooling duct. The heat will be taken by the coolant travelling at that point and hence there will be a rise in the temperature. This rise in the temperature can be detected by the sensors at the downstream. The coolant travel speed can be noted directly when measuring the time that passes between the detection of heat pulse by 2 sensors considering the distance between these sensors. In order to utilize this method, a high measurement resolution is needed. The data extraction from the electromechanical sensors from a rotating rotor may not be easy. The possible techniques that can be employed are use of slip rings or telemetry.

The problems that one will encounter by using slip rings are the electrical noise that will be generated by sliding brush can corrupt the data being collected. Apart from that there is a limitation in the possible number of simultaneous channel of data because of the cross talk noise and due to the fact that the slip rings cannot be too large. Heavy weight is also a notable disadvantage of slip rings. On other hand, telemetry could be an alternate choice but still the telemetry is prone to electromagnetic interferences. The survival, implementation and operation of electronic system in such a low temperature are doubtful.

Alternatively, an optical measurement system based on fiber Bragg grating (FBG) principle can be a solution. FBG sensors are having numerous advantages compared to electromechanical sensors like possibility of wavelength division multiplexing (WDM) and hence many sensors could be multiplexed in a single glass fiber. Another advantage relevant to the HTS generator application is the signal transmission. The signal from the sensor could be easily transmitted from a rotating machine using a vacuum tight rotatory optical feed through (VROF) and this system is immune to electromagnetic interferences.

In this paper, the initial experiments, which demonstrate the rotational effect on FBG sensor at room temperature is reported.

\section{FBG Principle}

FBG principle is well documented in many literatures [4], which works on the principle of wavelength reflection due to the change in the grating length. The general measurement scheme and the structure of the sensor are shown in figure 2.

Gratings with different spatial period are arranged at various positions along a single mode fiber in the HTS Generator. Rotor temperature will vary the gratings' periods, which can be read out with a tunable laser in a wavelength division multiplexing (WDM) scheme. The spectral position of the reflections may be correlated with the spatial position of the gratings, and the shift of the gratings' maximum reflection indicates the change of the 
gratings' period, which in turn measures temperature

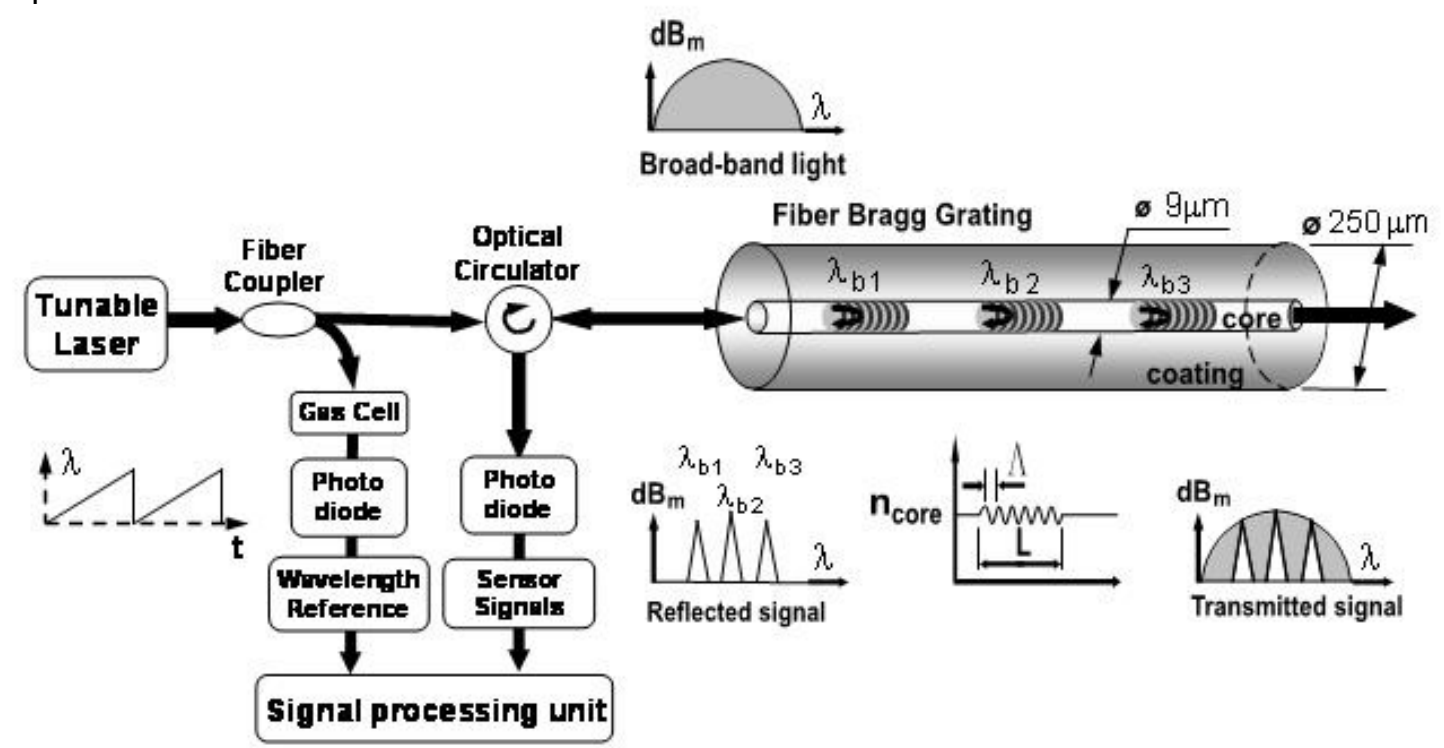

Fig. 2. General Measurement scheme of Fibre Bragg Gratings

\section{Signal integration and Processing}

The block diagram of the interrogation unit used in this study is shown in figure 2. Light from the tunable laser was split into two by a 50/50 fibre coupler and optical circulator. Half of the light was guided to a 10-FBG-sensor array while the other half to a National Institute of Standards and Technology traceable wavelength gas cell. This gas cell covers the wavelength range from $1520 \mathrm{~nm}$ to $1570 \mathrm{~nm}$. When the wavelength of light emitted by the laser is continuously swept from $1520 \mathrm{~nm}$ to $1570 \mathrm{~nm}$, reflections from FBGs are obtained at the photo detector at different instants. A centroid method is then used to determine the central wavelength of the FBGs. Table 1 shows the specification of the detection unit

Tab 1: Specification of the detection unit

\begin{tabular}{|l|l|}
\hline Technical parameter & Specification \\
\hline Resolution & $0.5 \mathrm{pm}$ \\
\hline Absolute accuracy & $\pm 2.0 \mathrm{pm}$ \\
\hline Repeatability & $\pm 1.0 \mathrm{pm}$ \\
\hline Optical output power & $10 \mathrm{dBm}$ \\
\hline Line width & $125 \mathrm{kHz}$ \\
\hline Optical Isolation & $45 \mathrm{~dB}$ \\
\hline
\end{tabular}

\section{Experimental setup}

In order to use the FBG sensors in a HTS generator application, it is important to check the survival of FBG sensors at high speed rotating devices and to estimate the associated error induced due to the high rotational speed. The VROF has to be validated for its signal transmission capability without any signal distortion and for the capacity of handling 3600 rpm rotational speed.

To investigate the above discussed issues, a simple experimental setup was developed at ITEP which is shown in Figure 3.

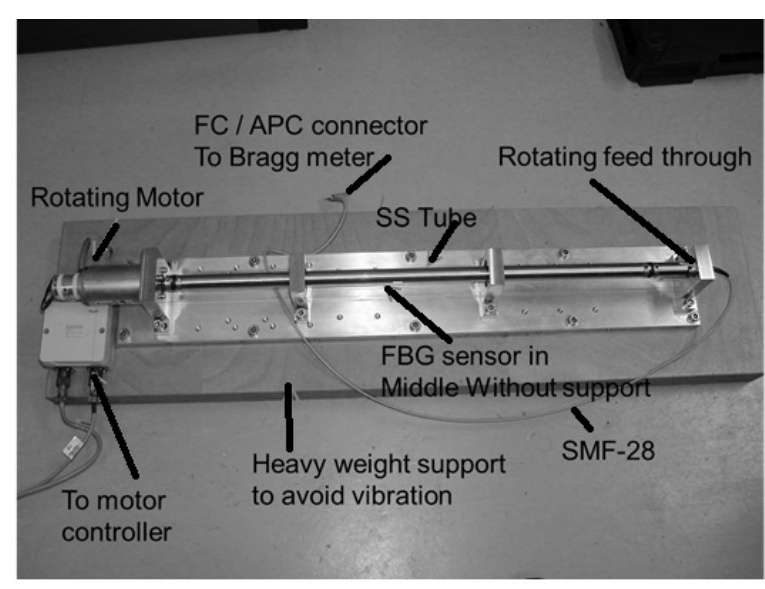

Fig 3 Setup to study the rotational effects of FBG sensors

The whole setup was built on top of heavy wooden block to avoid any vibration in the system during rotation which may affect the FBG sensor signal. One end of the stainless steel tube (SST) was connected to the rotating motor which was controlled by a $0-10 \mathrm{~V}$ power supply. Increase in the voltage increase the rotating speed of the motor and hence the SST. The other end of the SST was connected to the VROF. The middle portion of the SST was held by 2 supports allowing the tube to rotate freely. The support is to ensure that there is no wobbling effect in the SST during its operation. 
The FBG sensor which was fabricated in single mode fiber type SMF-28 was installed in the middle of the tube as shown in figure 4.

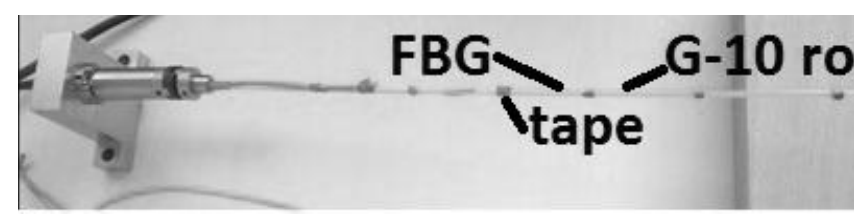

Fig 4 Sensor Installations.

The sensor was taped along with G-10 fiber rod to avoid any bending of the fiber and to provide mechanical support during SST rotation. The stator of the VROF was connected to the detection unit.

\section{Results and discussion}

The experimentation was then conducted by slowing increasing the driving voltage of the rotating motor and hence the rotating speed of the SST to $3600 \mathrm{rpm}$. The sensor response was continuously monitored and recorded. Table 2 gives the operating sequence of the experimentation.

Tab 2: Experiment operating sequence

\begin{tabular}{|c|c|c|}
\hline Sequence & Pattern & $\begin{array}{c}\text { Rotating } \\
\text { speed }\end{array}$ \\
\hline 1 & $\begin{array}{c}\text { Increasing } \\
\text { Ramp up }\end{array}$ & $0-3600 \mathrm{rpm}$ \\
\hline 2 & $\begin{array}{c}\text { Decreasing } \\
\text { Ramp down }\end{array}$ & $3600-0 \mathrm{rpm}$ \\
\hline 3 & $\begin{array}{c}\text { Constant } \\
\text { rotation }\end{array}$ & $10 \mathrm{~min}$ at \\
& $\begin{array}{c}\text { Pulse ramp up } \\
\text { and ramp } \\
\text { down }\end{array}$ & $\begin{array}{c}0-3600 \mathrm{rpm} \\
\text { ramp up and } \\
\text { ramp down }\end{array}$ \\
\hline 4
\end{tabular}

From the figure $5(a-d)$, it could be observed that for various operating sequences the Bragg wavelength shift was found to be $\pm 6 \mathrm{pm}$. The amount of error contribution due to the rotation has been estimated for various metal and polymer coated FBG sensor. The fabrication, temperature calibration of these sensors has been reported by the authors in their previous publications $[5,6]$.

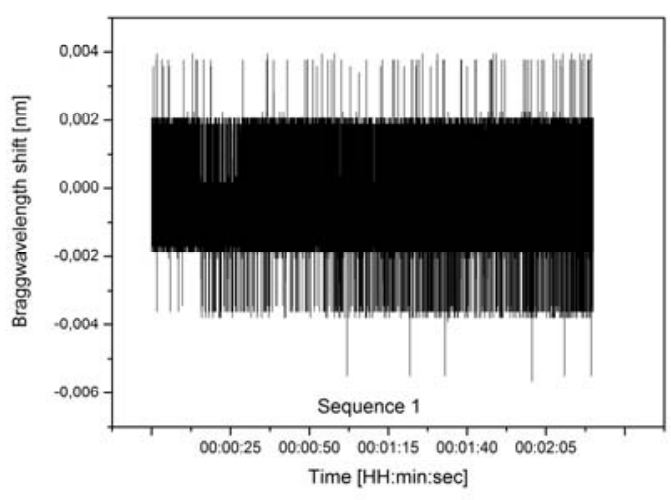

Fig 5a. Increasing ramping up (Sequence 1)

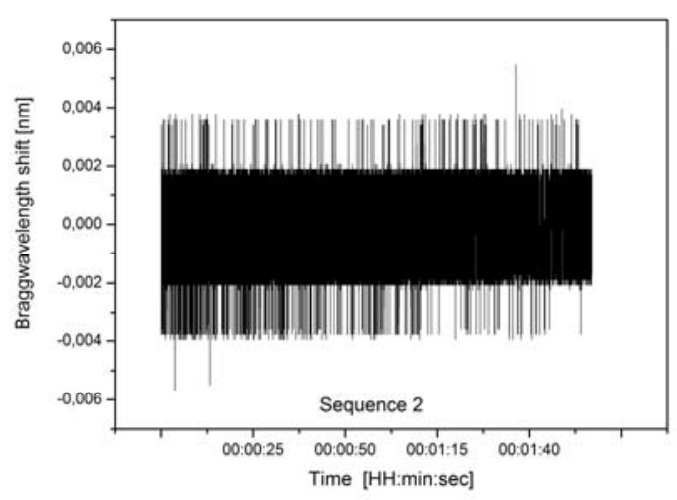

Fig 5b. Decreasing ramping down (Sequence 2)

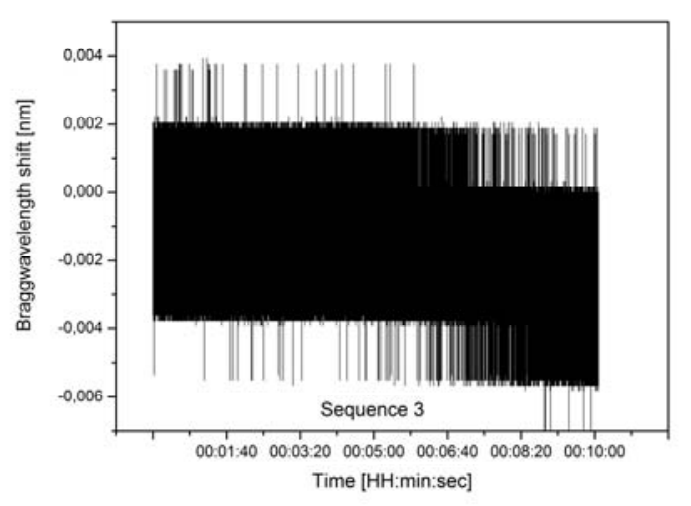

Fig 5c. Constant rotation (Sequence 3)

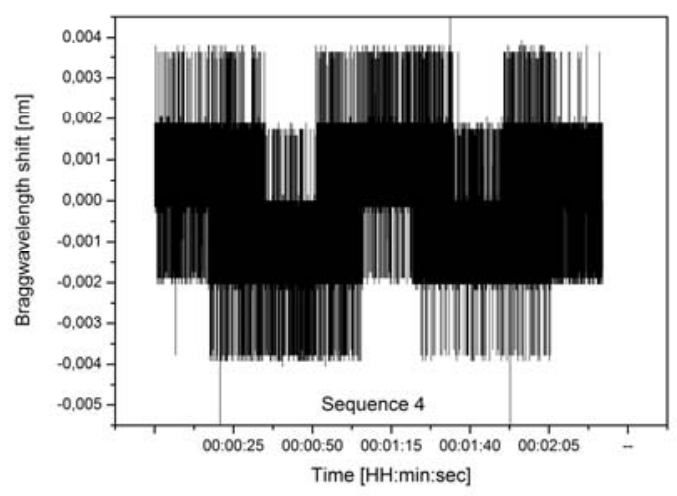

Fig 5d: Pulse ramp up and ramp down (Sequence 4) 
The estimated temperature sensitivity at $30 \mathrm{~K}$ and the corresponding temperature error due to the rotation has been shown in table 3 . It can be seen that the error due to the rotational effect becomes small for higher temperature sensitivity sensors. Moreover, the Bragg wavelength shift for all sequence of operation lies within $\pm 6 \mathrm{pm}$ and hence it could be corrected during the signal processing.

Tab 3: Estimated rotational error for various sensors

\begin{tabular}{|c|c|c|c|}
\hline $\begin{array}{l}\text { Sensor } \\
\text { type }\end{array}$ & $\begin{array}{c}\text { Sensitivity } \\
@ 30 \mathrm{~K} \\
{[\mathrm{pm}]}\end{array}$ & $\begin{array}{c}\text { Shift } \\
\text { due to } \\
\text { Rotation } \\
\text { [pm] }\end{array}$ & $\begin{array}{c}\text { Estimated } \\
\text { Error due } \\
\text { to rotation } \\
\quad[\mathrm{K}]\end{array}$ \\
\hline $\begin{array}{c}\text { Acrylate } \\
\text { FBG }\end{array}$ & $6 \mathrm{pm}$ & $\pm 6 \mathrm{pm}$ & $\pm 1 \mathrm{~K}$ \\
\hline $\begin{array}{l}\text { Ormocer } \\
\text { FBG }\end{array}$ & $8 p m$ & $\pm 6 \mathrm{pm}$ & $\pm 0,75 \mathrm{~K}$ \\
\hline $\begin{array}{c}\text { Aluminium } \\
\text { Embedded } \\
\text { FBG }\end{array}$ & 22 pm & $\pm 6 \mathrm{pm}$ & $\pm 0,272 \mathrm{~K}$ \\
\hline $\begin{array}{c}\text { Copper } \\
\text { Embedded } \\
\text { FBG }\end{array}$ & 18 pm & $\pm 6 \mathrm{pm}$ & $\pm 0,333 \mathrm{~K}$ \\
\hline $\begin{array}{l}\text { Lead } \\
\text { Embedded } \\
\text { FBG }\end{array}$ & 33 pm & $\pm 6 \mathrm{pm}$ & $\pm 0,181 \mathrm{~K}$ \\
\hline $\begin{array}{c}\text { Indium } \\
\text { Embedded } \\
\text { FBG }\end{array}$ & 33 pm & $\pm 6 \mathrm{pm}$ & $\pm 0,181 \mathrm{~K}$ \\
\hline $\begin{array}{l}\text { Copper } \\
\text { coated } \\
\text { FBG }\end{array}$ & 24 pm & $\pm 6 \mathrm{pm}$ & $\pm 0,25 \mathrm{~K}$ \\
\hline
\end{tabular}

\section{Conclusion}

From the above experimental results and the estimated error values, it can be concluded that the FBG sensors can survive high speed rotation without getting damaged. Also, the error estimated due to the rotation of such sensors lies within $\pm 6 \mathrm{pm}$ and this could be easily corrected during signal processing. Apart from that, the VROF was able to transmit the signal without any distortion and signal losses. Hence FBG sensors could be an alternate choice for HTS generator application, provided it satisfies all other measurement requirements.

\section{Acknowledgement}

This work was supported by German Federal Ministry of Economics and Technology.

\section{References:}

[1].Dr. Jörn Grundmann - Entwicklung roterierender HTS Machinen bei Siemens ,Vortrag, Mai 2008 (https://www.tu-braunschweig.de/Medien-

DB/iot/vortrag-grundmann.pdf)

[2].A. Hofmann, Self-regulating transfer modes of liquid helium to the rotor of a superconducting generator, Cryogenics 21: 372 (1981)

[3].Method and apparatus for replenishing the helium bath in the rotor of a superconducting generator, Patent 4329849 Issued on May 18, 1982

[4].Othonos A, Kalli K, "Fiber Bragg Gratings Fundamentals and application in telecommunications and sensing", Artech House optoelectronics library, 1999

[5].Rajini-Kumar R, Suesser M, Narayankhedkar K G, Krieg G, Atrey M D. "Design parameter evaluation of a metal recoated Fiber Bragg Grating sensors for measurement of cryogenic temperature or stress in superconducting devices"- Cryogenics 49 (2009) 202-209

[6].Rajini-Kumar R, Suesser M, Narayankhedkar $K$ G, Krieg G, Atrey M D,"Performance evaluation of metallic coated Fiber Bragg Grating sensors for sensing cryogenic temperature"- cryogenics, Vol 48/3-4 pp 142-147 2008. 